complete right bundle branch block (CRBBB), atrioventricular block (AV block), infarct or pseudounfarct, ST depression.

Results Electrocardiographic alterations were found in $65 \%$ of SSc patients, but only in $18,19 \%$ of RA patients. The difference was significant $(\mathrm{p}<0,001)$ Table 1 . In the dSSc group, ECG alterations were found in 6 pts (15\%)- not significant compared to the 1SSc group (5 pts, 12,5\%). p = o,220. ST depression was found in 2 pts $(5 \%)$ with dSSc, but only in 1 pts $(4,5 \%)$ with RA. Infarct or pseudoinfarct were found in 9 pts $(22,5 \%)$ with $\mathrm{SSc}(6$ in $\mathrm{dSSc}, 3$ in $1 \mathrm{SSc})$.

\begin{tabular}{lllll}
\multicolumn{4}{l}{ Abstract FRI0200 Table 1} \\
\hline RA & $\%$ & SSC & $\%$ \\
\hline VPB & 3 & 13,64 & 10 & 25 \\
SVPB & 1 & 4,55 & 4 & 10 \\
Ar. Abs. & - & - & 3 & 7,5 \\
LBBB & - & - & 1 & 2,5 \\
LAFB & - & - & 2 & 5,0 \\
CRBBB & - & - & 3 & 7,5 \\
AV Block & - & - & 3 & 7,5 \\
\hline
\end{tabular}

Electrocardiographic alterations in patients with SSC and RA.

Conclusion The results of our study showed a significant prevalence of ECG alterations in SSc, but low prevalence in RA. Differences between a presence of alterations in two subgroup $(\mathrm{dSSc}, 1 \mathrm{SSc})$ of SSc is not significant $(\mathrm{p}=0,220)$.

\section{FRI0201 CIRCULATING PROCOLLAGEN III AMINOPROPEPTIDE IN SYSTEMIC SCLEROSIS: RELATIONSHIP TO A RECENTLY VALIDATED DISEASE ACTIVITY INDEX}

${ }^{1} S$ D'Angelo, ${ }^{2} \mathrm{~A}$ Della Rossa, ${ }^{3} \mathrm{M}$ Caronni, ${ }^{3} \mathrm{R}$ Scorza, ${ }^{2} \mathrm{~S}$ Bombardieri, ${ }^{1} \mathrm{G}$ Valentini. ${ }^{1}$ Rheumatology Unit, Second University of Naples, Naples; ${ }^{2}$ Clinical Immunology Unit, University of Pisa, Pisa; ${ }^{3}$ Clinical Immunology Unit, University of Milan, Milan, Italy

\subsection{6/annrheumdis-2001.282}

Background Systemic sclerosis (SSc) is characterised by excessive deposition of extracellular matrix components in the skin and in involved internal organs. Serum levels of procollagen III aminopropeptide (PIIINP), an index of collagen synthesis, have been reported as a possible marker of disease activity in SSc. ${ }^{1}$

Objectives To study the relationship between a recently developed index of disease activity $(2,3)$ and serum concentrations of PIIINP.

Methods Nineteen SSc patients (17 F, 2 M; age ranging from 24 to 76 , median 55.5 years; $13 \mathrm{lcSSc}, 6 \mathrm{dcSSc}$; disease duration ranging from 2 to 34 , median 12 years) consecutively admitted to 3 Italian tertiary centres and 19 sex and age-matched healthy controls were investigated, for serum levels of PIIINP, by radioimmunoassay.

For each SSc patient a recently validated activity index ${ }^{2,3}$ was calculated.

Results Increased serum concentrations of PIIINP were found in SSc compared with controls $(4.59 \pm 1.84$ vs. $3.05 \pm 0.85$ micrograms $/ \mathrm{L} ; \mathrm{p}=0.002)$. Age, sex, subset and disease duration did not influence PIIINP serum levels.

The activity scores calculated in SSc patients ranged from 0.0 to 7.0, median 2.0. No significant correlations were registered between the PIIINP levels and the calculated activity index (rho $=0.04 ; \mathrm{p}>0.05)$.
Conclusion This study demonstrates that elevated PIIINP serum levels found in SSc, even reflecting fibroblast activation, cannot be used as a surrogate for the measurement of disease activity as evaluated by a recently proposed index.

\section{REFERENCES}

1 Stevens, et al. Br J Rheumatol. 1992;31:683-89

2 Valentini, et al. Arthritis Rheum. 2000;43:S9-S392

3 Valentini, et al. Ann Rheum Dis. 2001, in press

\section{FRI0202 LOW-DOSE ORAL CYCLOPHOSPHAMIDE IN THE TREATMENT OF "ACTIVE" SYSTEMIC SCLEROSIS: AN OPEN PROSPECTIVE 2-YEAR STUDY}

${ }^{1} \mathrm{G}$ Cuomo, 'S D'Angelo, ${ }^{1} \mathrm{C}$ Naclerio, ${ }^{2} \mathrm{M}$ Menegozzo, ${ }^{1} \mathrm{G}$ Valentini. ${ }^{1}$ Rheumatology Unit; ${ }^{2}$ Professional Immunopathology Unit, Second University of Naples, Naples, Italy

10.1136/annrheumdis-2001.283

Background Previous studies have reported cyclophosphamide (CYC) to be active in the treatment of fibrosing alveolitis in its early, inflammatory stages. Akesson et al. ${ }^{1}$ demonstrated, in CYC treated SSc patients with alveolitis, an effect on inflammation parameters and skin thickening.

Objectives To test, by a prospective 2-years trial, the efficacy and safety of low-dose oral CYC in the treatment of ?active? SSc whatever the disease manifestation underlying activity.

Methods SSc patients consecutively admitted were divided into 2 groups: group I with active disease (i.e. patients who had a new disease manifestation and/or a deterioration of a previously present one in the six months preceding the enrollment or who presented laboratory alterations indicating immune-inflammatory activity such as hypergammaglobulinemia and/or hypocomplementemia) and group II with inactive disease (i.e. patients with neither feature). Group I patients were treated with oral CYC $(50 \mathrm{mg} /$ daily $)$ in addition to the previously administered symptomatic treatment; group II patients were only treated with symptomatic therapy. The following parameters (total skin score; ulcer healing; FVC; DLCO; EF; gammaglobulin levels; complement levels) and the severity scales recently proposed by Medsger et $a l .^{2}$ were carried out at 12 and 24 months.

Results Out of the 45 recruited patients ( $42 \mathrm{~F}, 3 \mathrm{M}$; aged 30 to 72 years, median age $49 ; 34$ with lcSSc, 11 with dcSSc; disease duration ranging from 1 to 34 , median 16 years) 22 were included in group I and 23 in group II. The study was discontinued for lack of efficacy in 5 out of the 19 group I patients and 8 out of the 19 group II patients who had at least the 12-months assessment $(\mathrm{p}>0.05)$. No significant difference was detected between the 2 groups in any clinical, laboratory and instrumental parameters assessed, including Medsger?s severity scales. C3 and C4 significantly increased in the group I patients after 12 and 24 months; ESR decreased in group I patients only after 12 months.

Conclusion Our results suggest that CYC might be able to contrast the evolution of disease in a significant percentage of SSc active patients. The role as a disease-modifying drug of SSc as a whole must be further investigated.

\section{REFERENCES}

1 Akesson, et al. Arthritis Rheum. 1994;37:729-35

2 Medsger TA, et al. J Rheumatol. 1999:26:2159-67 


\section{FRI0203 PULMONARY INVOLVEMENT IN JUVENILE SCLERODERMA}

AM Romicka, A Malinowski. Pediatric Clinic, Institute of Rheumatology, Warsaw, Poland

10.1136/annrheumdis-2001.284

Background Scleroderma in children manifests more frequently in the form of localised disease than syptemic one. This disorder is characterised by the presence of hard and tight skin (linear, localised and generalised morphea); systemic sclerosis, besides the skin changes it manifests the internal organ involvement.

Objectives The aim of this report was an assessment of the frequency and nature of lung lesions in children with scleroderma. Methods The study comprised 32 non selected pts, aged from 4 to 18 yrs., 16 suffering from localised and 16 from systemic scleroderma. The disease duration ranged from 6 months to $14 \mathrm{yrs}$. The relation between clinical symptoms, $\mathrm{x}-$ ray findings and functional pulmonary tests were performed.

Results Clinical and/or radiological signs (cought, dyspnoe, interstitial thickening) were found in 9 pts. The results of the functional investigation showed an impairment of the pulmonary function in $80.6 \%$ of pts with systemic sclerosis and $50 \%$ - with localised scleroderma, (total 65.6\%). A decrease in DLCO (in 1/ 3 of pts in association with restrictive ventilatory failure) belonged to the most frequent of the lung damages.

Conclusion

- Pulmonary involvement is frequent, however not a main feature in the course juvenile scleroderma.

- Abnormalities in functional tests were often the only manifestation of the respiratory sys-tem involvement.

- Lung functional disturbances may be independent of clinical features, muscular on skin involvement.

- Respiratory functional tests are helpful in estimation of systemic scleroderma in the cases manifesting only the clinical form of localised one.

\section{REFERENCES}

1 Chomette G, Tranbaloc P, Beaufils H, et al. Les sclerodermies viscerales. Ann Med Interne (Paris) 1984;135:606

2 Greenwald Gl, Tashkin DP, Gong $\mathrm{H}$, et al. Longitudinal changes in lung function and respiratory symptoms in progressive systemic sclerosis. Am J Med. 1987;83:83

3 Lahman TJA. Systemic and localized scleroderma in children. Curr Opin Rheumatol. 1995;8:576

4 Uziel Y, Miller H, Lazar RM. Scleroderma in children. Pediatr Clin North Am. 1995:42:1171

5 Deheu I, Roujcan J-C, Cosnes A. Revuz: internal involvement in localized scleroderma. Medicine (Baltimore) 1994:73:241

6 Laxer RM, Feldman BM. General and local scleroderma in children and dermatomyositis and associated syndromes. Curr Opin Rheumatol. 1997;9:458

\section{FRI0204 TRIGEMINAL SENSORY NEUROPATHY IN SYSTEMIC SCLEROSIS}

YV Grachov, NG Guseva, VM Grinin, ML Kukushkin, AV Syrovegin, VF Guravlev. Clinical Department, Institute of Rheumatology of RAMS, Moscow, Russia

\subsection{6/annrheumdis-2001.285}

\section{Background}

Objectives Systemic sclerosis (SSc) is one of the few somatic disorders which induce isolated or combined trigeminal sensory neuropathy (TSN).

Methods A screening of 101 patients with SSc hospitalised at the Institute of Rheumatology over a period of six months in 2000 revealed that eight patients $(7,9 \%)$ had TSN (all of them were females aged 42-69 with a clinical history of SSc of 1 to
12 years). In two cases, SSc was associated with the SJÖGREN'S syndrome; one case was associated with polymyositis. All the patients with TSN underwent rheumatological and neurological examinations including the recording of blink reflex.

Results The main clinical manifestation of TSN was bilateral asymmetric numbness in all the three divisions of the trigeminal nerve, especially in the perioral area. Five patients had qualitative taste disorders including «oral astereognosis», i.e., monotypical sensations in the oral cavity on taking up foods of different kinds and tastes. In two cases, TSN was manifested as constant and constant or «neuralgia-like» facial pains. Irrespective of remissions (which could be spontaneous), in each case facial pain was one of the most significant manifestations of SSc. All the patients had disturbances in surface sensitivity (hyper- or hypoesthesia) in the distal parts of the upper and lower extremities. In three cases, early symptomes of TSN (e.g., facial paraesthesia, numbness and hyperesthesia) developed simultaneously with the changes in the skin colour (especially of fingers), skin oedema, arthralgias and malaise. In the rest of the patients, TSN developed within 6 months to 3 years after the Raynaud's syndrome, sclerodactyly and other manifestations of SSc. The blink reflex records revealed modest prolongation of ipsilateral R1 responses. Specific therapy of TSN included administration of vasoactive drugs (e.g., pentoxifylline, vazaprostan) and group B vitamins. The elimination of facial pain presented a problem. The use of NSAID resulted in short-term attenuation of pain; in one case, topical application of ibuprofen (in the form of a cream) was more effective. Patients with paroxysmal manifestations of pain received additionally carbamazepine $(300-400 \mathrm{mg} / \mathrm{d})$. The efficiency of physio- and reflexotherapy in patients with acute facial pain syndrome is open to question.

Conclusion TSN is a typical neurological sign of SSc (with frequency $7,9 \%$ of cases), which is manifested as facial numbness, qualitative taste disorders including "oral astereognosis», pain and paraesthesiae. Most often, TSN develops within 6 months to 3 years after the appearance of other characteristic features of SSc. A combination of bilateral sensory disturbances in the facial area and distal parts of the upper and lower extremities suggests that TSN can be regarded as a partial form of generalised sensory polyneuropathy.

\section{FRI0205 CLUES TO SYSTEMIC SCLEROSIS SINE SCLERODERMA}

${ }^{1}$ I Rosner, ${ }^{2} \mathrm{G}$ Slobodin, ${ }^{1} \mathrm{M}$ Rozenbaum, ${ }^{2} \mathrm{JE}$ Naschitz, ${ }^{3} \mathrm{E}$ Toubi, ${ }^{3} \mathrm{~A}$ Kessel, ${ }^{2} \mathrm{D}$ Yeshurun. ${ }^{1}$ Rheumatology; ${ }^{2}$ Internal Medicine A; ${ }^{3}$ Immunology, Bnai Zion Medical Center, Haifa, Israel

10.1136/annrheumdis-2001.286

Background There is substantial difficulty in the diagnosis of progressive systemic sclerosis where visceral involvement precedes cutaneous expression of the disease.

Objectives To describe clues to the diagnosis of progressive systemic sclerosis sine scleroderma (ssSSc).

Methods Report of 3 cases and review of the MEDLINE database concerning ssSSc.

Results Tweny five patients from the literature and 3 from our unit fulfilled the inclusion criteria, 13 females and 15 males, mean age 51.2(SD17.2) years. Conditions motivating the search for ssSSc were pulmonary fibrosis (44\%), congestive heart failure (19\%), esophageal dysfunction (7\%), malabsorption (11\%), nephropathy (11\%), fever of unknown origin $(3.5 \%)$ and seizures $(3.5 \%)$. An overt undifferentiated connective tissue disease was present in 7 pts. Multisystem involvement became apparent 